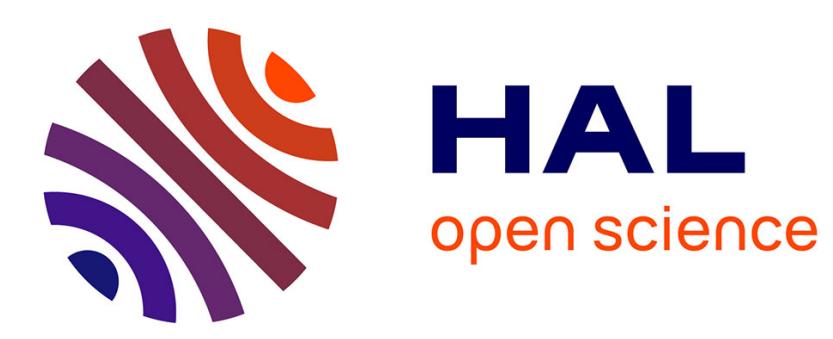

\title{
POSSIBILITÉS D'EXPÉRIENCES AU CYCLOTRON DU CNRS A ORLÉANS
}

\author{
$\mathrm{Ph}$. Albert
}

\section{To cite this version:}

Ph. Albert. POSSIBILITÉS D'EXPÉRIENCES AU CYCLOTRON DU CNRS A ORLÉANS. Journal de Physique Colloques, 1973, 34 (C5), pp.C5-77-C5-77. 10.1051/jphyscol:1973514 . jpa-00215301

\section{HAL Id: jpa-00215301 https://hal.science/jpa-00215301}

Submitted on 1 Jan 1973

HAL is a multi-disciplinary open access archive for the deposit and dissemination of scientific research documents, whether they are published or not. The documents may come from teaching and research institutions in France or abroad, or from public or private research centers.
L'archive ouverte pluridisciplinaire HAL, est destinée au dépôt et à la diffusion de documents scientifiques de niveau recherche, publiés ou non, émanant des établissements d'enseignement et de recherche français ou étrangers, des laboratoires publics ou privés. 


\title{
POSSIBILITÉS D'EXPÉRIENCES AU CYCLOTRON DU CNRS A ORLÉANS
}

\author{
Ph. ALBERT
}

CNRS, Laboratoire du cyclotron, 45045 Orléans-Cédex, France

\begin{abstract}
Résumé. - La mise en service du cyclotron installé par le CNRS au Centre d'Orléans-la-Source est prévue en 1974. Ce cyclotron a été conçu spécialement pour la production de faisceaux intenses de particules chargées en vue d'études d'analyses par activation, de la réalisation d'expériences de physique, de chimie ou de biologie au moyen de radio-isotopes de périodes courtes et moyennes et de toutes les études concernant les phénomènes physiques et chimiques produits par l'irradiation. Ce cyclotron Thomson-CSF à énergie variable est de même conception que celui de l'ISN à Grenoble.
\end{abstract}

Les caractéristiques prévues sont les suivantes :

$$
\begin{gathered}
\text { protons de } 3 \text { à } 47 \mathrm{MeV} \\
\text { deutons de } 5 \text { à } 24 \mathrm{MeV} \\
\alpha \quad \text { de } 10 \text { à } 48 \mathrm{MeV} \\
3 \mathrm{He} \quad \text { de } 7 \text { à } 64 \mathrm{MeV} .
\end{gathered}
$$

Les intensités de faisceaux seront de 40 à $100 \mu \mathrm{A}$ sur les cibles externes.

Il est prévu de produire des faisceaux de neutrons rapides dans un large domaine d'énergie.

Il serait possible ultérieurement d'envisager l'accélération de tritons entre 7 et $16 \mathrm{MeV}$.

Une salle d'expérimentation dans laquelle les lignes de faisceaux seront installées permettra une grande souplesse d'aménagement des expériences puisque les diverses alvéoles seront séparées entre elles par des assemblages de blocs de bétons. Il sera donc possible d'en modifier facilement les dimensions en fonction des besoins.

Il est prévu pour les expériences des chimistes des irradiations sous ultra-vide cryogénique et le refroidissement des échantillons aux plus basses températures si cela est nécessaire.

Des laboratoires de chimie sont accolés à l'une des faces de la salle des irradiations pour faciliter la manipulation des radio-isotopes de très courtes périodes dans les délais les plus réduits.

Nous espérons que cet outil conçu pour être disponible pour les expériences des chimistes, physiciens et biologistes permettra à de nombreux chercheurs d'effectuer des recherches nouvelles dans des domaines très variés.

Les chercheurs qui souhaitent réaliser des expériences au cyclotron d'Orléans sont priés de prendre contact dans les plus courts délais avec M. Ph. Albert.

Abstract. - Particles energies and external beams intensities are given for the CNRS Cyclotron in Orléans Center.

This cyclotron will be used mainly for chemical, biological and physical experiments. 